\title{
Decomposition of Solutions of the Cauchy Problem of a Quasi-Homogeneous Partial Differential Equation
}

\author{
by \\ Kunio ICHINOBE and Masatake MIYAKe
}

\begin{abstract}
We give a decomposition formula for the formal solution of the Cauchy problem for a quasi-homogeneous partial differential equation with constant coefficients in the twodimensional complex plane. The decomposition formula, Theorem 1.1, is given in a form associated with the factorization of the relevant operator which is similar to the decomposition of the solution of an ordinary differential equation with constant coefficients.
\end{abstract}

2010 Mathematics Subject Classification: Primary 35C10; Secondary 35E15.

Keywords: decomposition of solutions, Cauchy problem.

\section{§1. Introduction}

Let $p$ and $q$ be relatively prime positive integers, and $P \equiv P\left(\partial_{t}^{p}, \partial_{x}^{q}\right)$ be a quasihomogeneous partial differential operator with constant coefficients in the twodimensional complex plane $\mathbb{C}^{2}=\mathbb{C}_{t} \times \mathbb{C}_{x}$, where $\partial_{t}$ and $\partial_{x}$ denote differentiation in the usual sense. We assume that the following factorization of $P$ is given:

$$
P\left(\partial_{t}^{p}, \partial_{x}^{q}\right)=\prod_{j=1}^{\mu} P_{j}\left(\partial_{t}^{p}, \partial_{x}^{q}\right)^{\ell_{j}}, \quad P_{j}\left(\partial_{t}^{p}, \partial_{x}^{q}\right)=\partial_{t}^{p}-\alpha_{j} \partial_{x}^{q},
$$

where $\left\{\alpha_{j}\right\}$ are nonzero complex constants which are mutually different.

We put $L:=\sum_{j=1}^{\mu} \ell_{j}$, which represents the total number of factors of $P$, and assume that $L \geq 2$.

Communicated by M. Kashiwara. Received September 2, 2010.

K. Ichinobe: Department of Mathematics Education, Aichi University of Education, 1 Hirosawa, Igaya, Kariya City, Aichi Pref., 448-8542 Japan;

e-mail: ichinobe@auecc.aichi-edu.ac.jp

M. Miyake: Graduate School of Mathematics, Nagoya University, Nagoya, 464-8602 Japan;

e-mail: mmiyake@math.nagoya-u.ac.jp

(C) 2012 Research Institute for Mathematical Sciences, Kyoto University. All rights reserved. 
Let $U(t, x)=\sum_{k \geq 0} U_{k}(x) t^{k}$ be the formal solution of the following Cauchy problem:

$$
\left\{\begin{array}{l}
P\left(\partial_{t}^{p}, \partial_{x}^{q}\right) U(t, x)=0 \\
\partial_{t}^{k} U(0, x)=0 \quad(0 \leq k \leq p L-2) \\
\partial_{t}^{p L-1} U(0, x)=\varphi(x)
\end{array}\right.
$$

where the Cauchy data $\varphi(x)$ is holomorphic in a neighbourhood of the origin. When $q>p$, the formal solution $U(t, x)$ diverges in general.

The purpose of this paper is to prove a decomposition formula for $U(t, x)$ in terms of the formal series

$$
u_{m}(t, x)=\sum_{j \geq 0} \alpha_{m}^{j} \varphi^{(q j)}(x) \frac{t^{p j+p-1}}{(p j+p-1) !} \quad(1 \leq m \leq \mu),
$$

which is the formal solution of the Cauchy problem for the factor $P_{m}$ :

$$
\left\{\begin{array}{l}
P_{m} u \equiv\left(\partial_{t}^{p}-\alpha_{m} \partial_{x}^{q}\right) u(t, x)=0 \\
\partial_{t}^{k} u(0, x)=0 \quad(0 \leq k \leq p-2) \\
\partial_{t}^{p-1} u(0, x)=\varphi(x)
\end{array}\right.
$$

Our main result is as follows.

Main Theorem 1.1. Let $U(t, x)$ be the formal solution of the Cauchy problem (1.2). Then there are uniquely determined $L$ constants $\left\{c_{m n} ; 1 \leq m \leq \mu, 1 \leq n\right.$ $\left.\leq \ell_{m}\right\}$, which satisfy the relation (2.13) below, such that the following decomposition formula holds:

$$
U(t, x)=\sum_{m=1}^{\mu} \sum_{n=1}^{\ell_{m}} c_{m n} \partial_{t}^{(1-p L)+p(n-1)} \frac{\left[(1 / p) \delta_{t}\right]_{n-1}}{(n-1) !} \partial_{t}^{p(1-n)+(p-1)} u_{m}(t, x),
$$

where $\delta_{t}=t \partial_{t}$ denotes the Euler operator and $\left[(1 / p) \delta_{t}\right]_{n-1}$ is given by

$$
\left[\frac{1}{p} \delta_{t}\right]_{k}:= \begin{cases}\frac{1}{p} \delta_{t}\left(\frac{1}{p} \delta_{t}-1\right) \cdots\left(\frac{1}{p} \delta_{t}-k+1\right), & k \geq 1 \\ 1, & k=0 .\end{cases}
$$

Here $\partial_{t}^{k}$ denotes differentiation or integration if $k>0$ or $k<0$, respectively, and $\partial_{t}^{-1}=\int_{0}^{t}$.

Remark. The formal solutions in the above decomposition formula are divergent in general when $q>p$, and therefore the formula is only in the formal sense. But, if the divergent solution $U(t, x)$ is Borel summable in some direction in the $t$-plane, 
then each $u_{m}(t, x)$ is also Borel summable in the same direction, and the formula (1.5) holds between the Borel sums associated with the divergent solutions. (The details on Borel summability of divergent solutions of this kind can be found in the papers by M. Miyake, K. Ichinobe and others [2]-[7].) Moreover, by the study of K. Ichinobe [3,4] on the integral kernel for the Borel sum, the formula (1.5) does hold between the integral kernels for the Borel sums.

The motivation for the study in this paper comes from the decomposition formula for solutions of ordinary differential equations with constant coefficients, which is explained by the following simple equation. Consider the solution $u(t)$ of

$$
\left(\frac{d}{d t}-\alpha\right)\left(\frac{d}{d t}-\beta\right) u(t)=0, \quad u(0)=0, u^{\prime}(0)=1 .
$$

First, consider the case $\alpha \neq \beta$. Then the decomposition

$$
\mathrm{id}=\frac{1}{\alpha-\beta}\left\{\left(\frac{d}{d t}-\beta\right)-\left(\frac{d}{d t}-\alpha\right)\right\}
$$

implies

$$
u(t)=\frac{1}{\alpha-\beta}\left\{u_{\alpha}(t)-u_{\beta}(t)\right\}
$$

with $u_{\alpha}(t)=(d / d t-\beta) u(t)$ and $u_{\beta}(t)=(d / d t-\alpha) u(t)$. Since $(d / d t-\alpha) u_{\alpha}(t)=0$ and $(d / d t-\beta) u_{\beta}(t)=0$, we get $u_{\alpha}(t)=C_{1} e^{\alpha t}$ and $u_{\beta}(t)=C_{2} e^{\beta t}$ as general solutions, respectively. Now the initial conditions $\left\{u(0)=0, u^{\prime}(0)=1\right\}$ show that $C_{1}=C_{2}=1$, that is, $u_{\alpha}(t)=e^{\alpha t}$ and $u_{\beta}(t)=e^{\beta t}$, which satisfy $u_{\alpha}(0)=1$ and $u_{\beta}(0)=1$.

Next, in the case $\alpha=\beta$ in (1.7), by letting

$$
\lim _{\beta \rightarrow \alpha} \frac{u_{\alpha}(t)-u_{\beta}(t)}{\alpha-\beta}=t e^{\alpha t}
$$

we get the solution of (1.7).

This observation on decomposition of solutions can be found in S. Mizohata's book [8].

Next, we illustrate the idea of decomposition by a simple example. Let $\alpha$ and $\beta$ be different non-zero complex numbers, and consider the solution $U(t, x)$ of the Cauchy problem

$$
\left\{\begin{array}{l}
\left(\partial_{t}-\alpha \partial_{x}^{2}\right)\left(\partial_{t}-\beta \partial_{x}^{2}\right) U(t, x)=0 \\
U(0, x)=0, \quad \partial_{t} U(0, x)=\varphi(x) .
\end{array}\right.
$$

We notice the following decomposition of $\partial_{t}$ :

$$
\partial_{t}=\frac{1}{\alpha-\beta}\left\{\alpha\left(\partial_{t}-\beta \partial_{x}^{2}\right)-\beta\left(\partial_{t}-\alpha \partial_{x}^{2}\right)\right\}
$$


This implies

$$
\mathrm{id}=\frac{1}{\alpha-\beta}\left\{\alpha \partial_{t}^{-1}\left(\partial_{t}-\beta \partial_{x}^{2}\right)-\beta \partial_{t}^{-1}\left(\partial_{t}-\alpha \partial_{x}^{2}\right)\right\}
$$

since $\partial_{t}^{-1} \circ \partial_{t}=$ id for functions with $U(0, x)=0$. Then we have the following decomposition of $U(t, x)$ :

$$
U(t, x)=\frac{\alpha}{\alpha-\beta} \partial_{t}^{-1} u_{\alpha}(t, x)+\frac{\beta}{\beta-\alpha} \partial_{t}^{-1} u_{\beta}(t, x),
$$

where $u_{\alpha}(t, x):=\left(\partial_{t}-\beta \partial_{x}^{2}\right) U(t, x)$ is the formal solution of the Cauchy problem

$$
\left(\partial_{t}-\alpha \partial_{x}^{2}\right) u_{\alpha}(t, x)=0, \quad u_{\alpha}(0, x)\left(=\partial_{t} U(0, x)\right)=\varphi(x),
$$

and similarly for $u_{\beta}(t, x):=\left(\partial_{t}-\alpha \partial_{x}^{2}\right) U(t, x)$.

Example 1.2 (cf. Proposition 3.1). 1. The case $P=\prod_{j=1}^{\mu} P_{j}$, that is, $L=\mu$ :

$$
U(t, x)=\sum_{m=1}^{\mu} c_{m} \partial_{t}^{p-p \mu} u_{m}(t, x), \quad c_{m}=\frac{\alpha_{m}^{\mu-1}}{\prod_{1 \leq j \leq \mu, j \neq m}\left(\alpha_{m}-\alpha_{j}\right)} .
$$

2. The case $P=P_{1}^{n}$ (cf. Lemma 2.1):

$$
U(t, x)=\partial_{t}^{1-p} \frac{\left[(1 / p) \delta_{t}\right]_{n-1}}{(n-1) !} \partial_{t}^{p(1-n)+(p-1)} u_{1}(t, x) .
$$

3. The case $p=1$ (cf. Lemma 2.1): Since $\left[\delta_{t}\right]_{n-1}=t^{n-1} \partial_{t}^{n-1}$, we have

$$
U(t, x)=\sum_{m=1}^{\mu} \sum_{n=1}^{\ell_{m}} c_{m n} \partial_{t}^{n-L} \frac{t^{n-1}}{(n-1) !} u_{m}(t, x) .
$$

To end the introduction, we remark that the results of this paper are valid for formal solutions of operators in $\mathbb{C}_{t} \times \mathbb{C}_{x}^{d}$ of the form

$$
P\left(\partial_{t}, \partial_{x}\right)=\prod_{j=1}^{\mu}\left(\partial_{t}^{p}-\alpha_{j} p\left(\partial_{x}\right)\right)^{\ell_{j}}
$$

with mutually different nonzero constants $\left\{\alpha_{j}\right\}$ and $p\left(\partial_{x}\right)=\sum_{|\alpha| \leq q} p_{\alpha} \partial_{x}^{\alpha}$, where $x \in \mathbb{C}_{x}^{d}$. It is enough to replace $\partial_{x}^{q}$ by $p\left(\partial_{x}\right)$ in the following proofs.

\section{$\S 2$. Proof of Main Theorem}

As a first step, we shall prove the decomposition formula in the case where $\mu=1$. 
Lemma 2.1. Let $v^{[n]}(t, x)$ be the formal solution of the Cauchy problem

$$
\left\{\begin{array}{l}
P_{0}^{n} v(t, x) \equiv\left(\partial_{t}^{p}-\alpha \partial_{x}^{q}\right)^{n} v(t, x)=0, \\
\partial_{t}^{k} v(0, x)=0 \quad(0 \leq k \leq p n-2), \quad \partial_{t}^{p n-1} v(0, x)=\varphi(x) .
\end{array}\right.
$$

Then

$$
\begin{aligned}
v^{[n]}(t, x) & =\sum_{j \geq 0} \alpha^{j} \frac{(j+1)_{n-1}}{(n-1) !} \varphi^{(q j)}(x) \frac{t^{p j+p n-1}}{(p j+p n-1) !} \\
& =\partial_{t}^{1-p} \frac{\left[(1 / p) \delta_{t}\right]_{n-1}}{(n-1) !} \partial_{t}^{p(1-n)+(p-1)} v^{[1]}(t, x),
\end{aligned}
$$

where

$$
(j+1)_{n-1}= \begin{cases}(j+1)(j+2) \cdots(j+n-1), & n \geq 2, \\ 1 & n=1,\end{cases}
$$

and $v^{[1]}(t, x)$ is the formal solution of (2.1) for $n=1$.

In particular, when $p=1$ the formula (2.3) reduces to

$$
v^{[n]}(t, x)=\frac{t^{n-1}}{(n-1) !} v^{[1]}(t, x)
$$

Proof. The formula (2.5) is an immediate consequence of (2.3) in view of $\left[\delta_{t}\right]_{n-1}=$ $t^{n-1} \partial_{t}^{n-1}$. Hence we shall prove (2.2) and (2.3). We put

$$
P_{0}\left(\partial_{t}^{p}, \partial_{x}^{q}\right)^{n} \equiv\left(\partial_{t}^{p}-\alpha \partial_{x}^{q}\right)^{n}=\partial_{t}^{p n}-\sum_{k=1}^{n} a_{k} \partial_{t}^{p(n-k)} \partial_{x}^{q k}
$$

where ${ }_{n} C_{k} \alpha^{k}=(-1)^{k-1} a_{k}(1 \leq k \leq n)$. For the operator $P_{0}^{n}$, the following polynomial is called the characteristic polynomial:

$$
P_{0}(\lambda, 1)^{n} \equiv(\lambda-\alpha)^{n}=\lambda^{n}-\sum_{k=1}^{n} a_{k} \lambda^{n-k}
$$

By a careful calculation, we see that

$$
v^{[n]}(t, x)=\sum_{j=0}^{\infty} A(j) \varphi^{(q j)}(x) \frac{t^{p j+p n-1}}{(p j+p n-1) !},
$$

where the coefficients $\{A(j)\}_{j=0}^{\infty}$ satisfy the recurrence formula

$$
A(j+n)=\sum_{k=1}^{n} a_{k} A(j+n-k), \quad j=-n+1,-n+2, \ldots
$$


with the initial conditions

$$
A(0)=1, \quad A(j)=0 \quad(j<0) .
$$

The formula (2.2) will be proved once we show

$$
A(j)=\frac{(n)_{j}}{j !} \alpha^{j}=\frac{(j+1)_{n-1}}{(n-1) !} \alpha^{j} \quad(j \geq 0),
$$

where $(n)_{j}=n(n+1) \cdots(n+j-1)=\Gamma(n+j) / \Gamma(n)$.

For the proof, let $f(x)$ be the generating function of the sequence $\{A(j)\}$ defined by

$$
f(x):=\sum_{j=0}^{\infty} A(j) x^{j} .
$$

From the recurrence formula (2.6), we have

$$
\sum_{j=0}^{\infty} A(j+n) x^{j+n}=\sum_{k=1}^{n}\left\{a_{k} x^{k} \sum_{j=0}^{\infty} A(j+n-k) x^{j+n-k}\right\} .
$$

Hence,

$$
f(x)-\sum_{j=0}^{n-1} A(j) x^{j}=\sum_{k=1}^{n}\left\{a_{k} x^{k}\left(f(x)-\sum_{j=0}^{n-k-1} A(j) x^{j}\right)\right\},
$$

which implies

$$
f(x)=\frac{A(0)+\sum_{j=1}^{n-1} x^{j}\left\{A(j)-\sum_{k=1}^{j} a_{k} A(j-k)\right\}}{1-\sum_{k=1}^{n} a_{k} x^{k}} .
$$

By the recurrence formula (2.6) with the initial conditions (2.7) we have

$$
A(j)-\sum_{k=1}^{j} a_{k} A(j-k)=0, \quad j=1, \ldots, n-1 .
$$

Thus

$$
f(x)=\frac{1}{1-\sum_{k=1}^{n} a_{k} x^{k}}=\frac{1}{(1-\alpha x)^{n}} .
$$

Taking $0<\varepsilon<|\alpha|^{-1}$, we have

$$
\begin{aligned}
A(j) & =\frac{1}{2 \pi i} \oint_{|x|=\varepsilon} \frac{f(x)}{x^{j+1}} d x=\lim _{x \rightarrow 0} \frac{1}{j !} f^{(j)}(x) \\
& =\frac{n(n+1) \cdots(n+j-1)}{j !} \alpha^{j}=\frac{(j+1)_{n-1}}{(n-1) !} \alpha^{j}
\end{aligned}
$$

This proves $(2.2)$. 
For the proof of $(2.3)$, we recall that

$$
v^{[1]}(t, x)=\sum_{j=0}^{\infty} \alpha^{j} \varphi^{(q j)}(x) \frac{t^{p j+p-1}}{(p j+p-1) !} .
$$

We notice the following relations:

$$
\begin{aligned}
\frac{t^{p j+p-1}}{(p j+p-1) !} \stackrel{\partial_{t}^{p(1-n)+(p-1)}}{\longrightarrow} \frac{t^{p j+p n-p}}{(p j+p n-p) !} \stackrel{\left[(1 / p) \delta_{t}\right]_{n-1}}{\longrightarrow}(j+1)_{n-1} \frac{t^{p j+p n-p}}{(p j+p n-p) !} \\
\stackrel{\partial_{t}^{1-p}}{\longrightarrow}(j+1)_{n-1} \frac{t^{p j+p n-1}}{(p j+p n-1) !}
\end{aligned}
$$

By applying these relations to (2.2), we obtain (2.3).

Proof of Main Theorem 1.1. We recall that

$$
P=\prod_{j=1}^{\mu} P_{j}^{\ell_{j}}, \quad P_{j}=\partial_{t}^{p}-\alpha_{j} \partial_{x}^{q}
$$

First of all, we assume that we can choose $L$ constants $\left\{c_{m n} ; 1 \leq m \leq \mu, 1 \leq n\right.$ $\left.\leq \ell_{m}\right\}$ so that the following operator equality holds:

$$
\partial_{t}^{p(L-1)}=\sum_{m=1}^{\mu} \sum_{n=1}^{\ell_{m}} c_{m n} \partial_{t}^{p(n-1)} \prod_{j=1, j \neq m}^{\mu} P_{j}^{\ell_{j}} P_{m}^{\ell_{m}-n} .
$$

Indeed, the linear equations for $\left\{c_{m n}\right\}$ are obtained by comparing the coefficients of $\partial_{t}^{p(L-j)} \partial_{x}^{q j}$ on both sides of the above equality. The details will be studied in the following sections.

Let $U(t, x)$ be the formal solution of the Cauchy problem (1.2). Then

$$
\partial_{t}^{p(L-1)} U(t, x)=\sum_{m=1}^{\mu} \sum_{n=1}^{\ell_{m}} c_{m n} \partial_{t}^{p(n-1)} \prod_{j=1, j \neq m}^{\mu} P_{j}^{\ell_{j}} P_{m}^{\ell_{m}-n} U(t, x) .
$$

Now we put

$$
U_{m}^{[n]}(t, x):=\prod_{j=1, j \neq m}^{\mu} P_{j}^{\ell_{j}} P_{m}^{\ell_{m}-n} U(t, x) .
$$

Then $U_{m}^{[n]}(t, x)$ is the formal solution of the Cauchy problem

$$
\left\{\begin{array}{l}
P_{m}^{n} U_{m}^{[n]}(t, x)=0 \\
\partial_{t}^{k} U_{m}^{[n]}(0, x)=0 \quad(0 \leq k \leq p n-2) \\
\partial_{t}^{p n-1} U_{m}^{[n]}(0, x)=\varphi(x)
\end{array}\right.
$$


Indeed, $P_{m}^{n} U_{m}^{[n]}=P U$ and $U(t, x)$ is the formal solution of (1.2). Hence by Lemma 2.1 and since $U_{m}^{[1]}(t, x)=u_{m}(t, x)$, which is given by (1.3), we have

$$
\partial_{t}^{p(L-1)} U(t, x)=\sum_{m=1}^{\mu} \sum_{n=1}^{\ell_{m}} c_{m n} \partial_{t}^{p(n-1)} \partial_{t}^{1-p} \frac{\left[(1 / p) \delta_{t}\right]_{n-1}}{(n-1) !} \partial_{t}^{p(1-n)+(p-1)} u_{m}(t, x) .
$$

By applying $\partial_{t}^{p(1-L)}$ to both sides, we obtain the desired formula (1.5).

Hence, the proof of the main theorem is reduced to proving the formula (2.13).

\section{§3. Proof of (2.13)}

We prepare some notation. Let $\alpha=\left(\alpha_{1}, \ldots, \alpha_{\mu}\right) \in(\mathbb{C} \backslash\{0\})^{\mu}$ and define

$$
f[\alpha]:=\prod_{j=1}^{\mu} \alpha_{j}^{\ell_{j}}, \quad f_{m}^{[n]} \equiv f_{m}^{[n]}[\alpha]:=f[\alpha] / \alpha_{m}^{n} \quad\left(1 \leq m \leq \mu, 1 \leq n \leq \ell_{m}\right) .
$$

Moreover, write $\partial_{\alpha}:=\sum_{j=1}^{\mu} \partial_{\alpha_{j}}$ and

$$
\Delta_{\alpha}^{k}:=\frac{\partial_{\alpha}^{k}}{k !}=\sum_{\substack{0 \leq k_{1}, \ldots, k_{\mu} \leq k \\ k_{1}+\cdots+k_{\mu}=k}} \frac{1}{k_{1} ! \cdots k_{\mu} !} \partial_{\alpha_{1}}^{k_{1}} \cdots \partial_{\alpha_{\mu}}^{k_{\mu}}
$$

Then we have the following proposition.

Proposition 3.1. The $L$ constants $\left\{c_{m n}\right\}$ in (2.13) are determined as the solution of the system of linear equations

$$
\mathcal{A} \vec{c}=\vec{e},
$$

where $\mathcal{A}$ is an $L \times L$ matrix and $\vec{c}, \vec{e}$ are column $L$ vectors, defined by

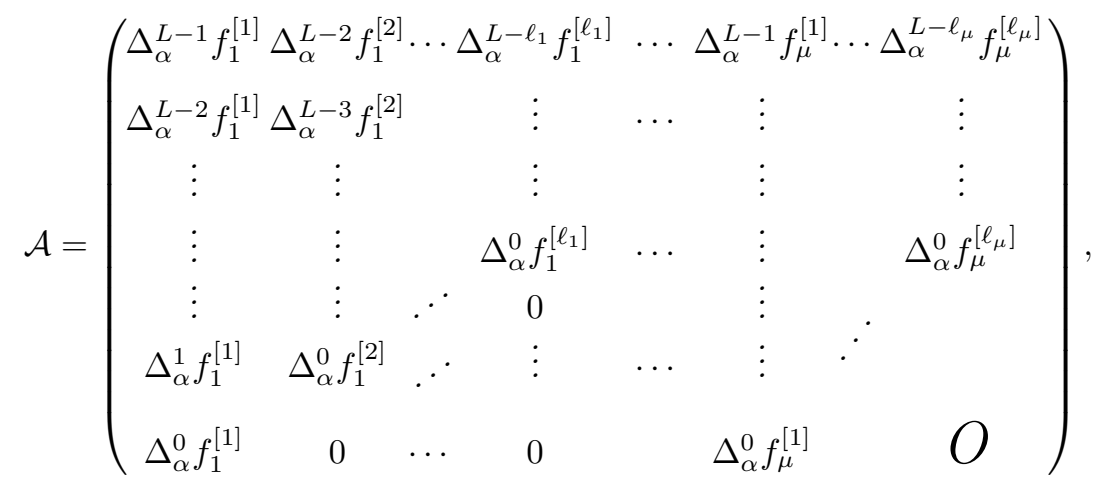

and

$$
\vec{c}={ }^{t}\left(c_{11}, \ldots, c_{1 \ell_{1}}, c_{21}, \cdots, c_{2 \ell_{2}}, \cdots, c_{\mu 1}, \ldots, c_{\mu \ell_{\mu}}\right), \quad \vec{e}={ }^{t}(1,0, \ldots, 0) .
$$


The unique existence of $\left\{c_{m n}\right\}$ is ensured by showing that $\operatorname{det} \mathcal{A} \neq 0$, which will be proved in the next section (cf. Lemma 3.3).

Example 3.2. 1. The case $P=\left(\partial_{t}^{p}-\alpha \partial_{x}^{q}\right)\left(\partial_{t}^{p}-\beta \partial_{x}^{q}\right)$ : Then $f[\alpha, \beta]=\alpha \beta$ and

$$
\mathcal{A} \vec{c}=\left(\begin{array}{ll}
1 & 1 \\
\beta & \alpha
\end{array}\right)\left(\begin{array}{l}
c_{11} \\
c_{21}
\end{array}\right)=\left(\begin{array}{l}
1 \\
0
\end{array}\right), \quad\left(\begin{array}{l}
c_{11} \\
c_{21}
\end{array}\right)=\frac{1}{\alpha-\beta}\left(\begin{array}{c}
\alpha \\
-\beta
\end{array}\right) .
$$

2. The case $P=\left(\partial_{t}^{p}-\alpha \partial_{x}^{q}\right)^{2}\left(\partial_{t}^{p}-\beta \partial_{x}^{q}\right)$ : Then $f[\alpha, \beta]=\alpha^{2} \beta$ and

$$
\mathcal{A} \vec{c}=\left(\begin{array}{ccc}
1 & 1 & 1 \\
\alpha+\beta & \beta & 2 \alpha \\
\alpha \beta & 0 & \alpha^{2}
\end{array}\right)\left(\begin{array}{c}
c_{11} \\
c_{12} \\
c_{21}
\end{array}\right)=\left(\begin{array}{l}
1 \\
0 \\
0
\end{array}\right), \quad\left(\begin{array}{c}
c_{11} \\
c_{12} \\
c_{21}
\end{array}\right)=\frac{1}{(\alpha-\beta)^{2}}\left(\begin{array}{c}
-\alpha \beta \\
\alpha(\alpha-\beta) \\
\beta^{2}
\end{array}\right) .
$$

3. The case $P=\left(\partial_{t}^{q}-\alpha \partial_{x}^{q}\right)^{L}$ : Then $f[\alpha]=\alpha^{L}$ and

$$
\begin{gathered}
\mathcal{A} \vec{c}=\left(\begin{array}{ccccc}
1 & 1 & \ldots & \ldots & 1 \\
\vdots & \vdots & & . \cdot & \\
\frac{(L-1)(L-2)}{2 !} \alpha^{L-3} & (L-2) \alpha^{L-3} & . \cdot & & \\
(L-1) \alpha^{L-2} & \alpha^{L-2} & & & \\
\alpha^{L-1} & 0 & & & 0
\end{array}\right)\left(\begin{array}{c}
c_{11} \\
c_{12} \\
\vdots \\
c_{1 L}
\end{array}\right)=\left(\begin{array}{c}
1 \\
0 \\
\vdots \\
0
\end{array}\right), \\
c_{1 n}=0(1 \leq n \leq L-1), c_{1 L}=1 .
\end{gathered}
$$

Proof of Proposition 3.1. First, we prove that $\left\{c_{m n}\right\}$ are solutions of the linear equations (3.1). Since the operators $\partial_{t}^{p}$ and $\partial_{x}^{q}$ commute, by substituting $\left(\tau, 1,-\alpha_{j}\right)$ into $\left(\partial_{t}^{p}, \partial_{x}^{q}, \alpha_{j}\right)$ in $(2.13)$, we have

$$
\tau^{L-1}=\sum_{m=1}^{\mu} \sum_{n=1}^{\ell_{m}} c_{m n} \tau^{n-1} \prod_{j=1}^{\mu}\left(\tau+\alpha_{j}\right)^{\ell_{j}} /\left(\tau+\alpha_{m}\right)^{n} .
$$

For any polynomial $g[\alpha]=g\left[\alpha_{1}, \ldots, \alpha_{\mu}\right]$ we have

$$
g\left[\tau+\alpha_{1}, \ldots, \tau+\alpha_{\mu}\right]=\sum_{p \geq 0} \tau^{p} \Delta_{\alpha}^{p} g[\alpha] .
$$

Therefore

$$
\prod_{j=1}^{\mu}\left(\tau+\alpha_{j}\right)^{\ell_{j}} /\left(\tau+\alpha_{m}\right)^{n}=\sum_{p=0}^{L-n} \tau^{p} \Delta_{\alpha}^{p} f_{m}^{[n]}[\alpha]
$$


and by employing inner product, we have

$$
\begin{aligned}
\tau^{n-1} \sum_{p=0}^{L-n} \tau^{p} \Delta_{\alpha}^{p} f_{m}^{[n]}[\alpha] & =\tau^{n-1}\left(\tau^{L-n}, \ldots, \tau^{0}\right)\left(\begin{array}{c}
\Delta_{\alpha}^{L-n} f_{m}^{[n]} \\
\vdots \\
\Delta_{\alpha}^{0} f_{m}^{[n]}
\end{array}\right) \\
& =\left(\tau^{L-1}, \ldots, \tau^{n-1}\right)\left(\begin{array}{c}
\Delta_{\alpha}^{L-n} f_{m}^{[n]} \\
\vdots \\
\Delta_{\alpha}^{0} f_{m}^{[n]}
\end{array}\right)
\end{aligned}
$$

Hence

$$
\begin{aligned}
& \tau^{L-1}=\sum_{m=1}^{\mu}\left(\tau^{L-1}, \ldots, \tau^{0}\right)\left(\begin{array}{cccc}
\Delta_{\alpha}^{L-1} f_{m}^{[1]} \Delta_{\alpha}^{L-2} f_{m}^{[2]} \cdots & \Delta_{\alpha}^{L-\ell_{m}} f_{m}^{\left[\ell_{m}\right]} \\
\Delta_{\alpha}^{L-1} f_{m}^{[1]} \Delta_{\alpha}^{L-2} f_{m}^{[2]} & \vdots \\
\vdots & \vdots & & \Delta_{\alpha}^{0} f_{m}^{\left[\ell_{m}\right]} \\
\vdots & \vdots & . \cdot & \\
\Delta_{\alpha}^{1} f_{m}^{[1]} & \Delta_{\alpha}^{0} f_{m}^{[2]} & & \\
\Delta_{\alpha}^{0} f_{m}^{[1]} & & 0
\end{array}\right)\left(\begin{array}{c}
c_{m 1} \\
\vdots \\
c_{m \ell_{m}}
\end{array}\right) \\
& =: \sum_{m=1}^{\mu}\left(\tau^{L-1}, \ldots, \tau^{0}\right) \mathcal{A}_{m}\left[L, \ell_{m}\right] \vec{c}_{m}\left[\ell_{m}\right] .
\end{aligned}
$$

Hence we can rewrite (3.3) as

$$
\begin{aligned}
\tau^{L-1} & =\left(\tau^{L-1}, \ldots, \tau^{0}\right)\left(\mathcal{A}_{1}\left[L, \ell_{1}\right], \ldots, \mathcal{A}_{\mu}\left[L, \ell_{\mu}\right]\right)\left(\begin{array}{c}
\vec{c}_{1}\left[\ell_{1}\right] \\
\vdots \\
\vec{c}_{\mu}\left[\ell_{\mu}\right]
\end{array}\right) \\
& =\left(\tau^{L-1}, \ldots, \tau^{0}\right) \mathcal{A} \vec{c},
\end{aligned}
$$

which implies the system of linear equations (3.1) immediately.

The following lemma proves the unique solvability of (3.1).

Lemma 3.3. For the matrix $\mathcal{A}$ in Proposition 3.1, we have

$$
\operatorname{det} \mathcal{A}= \begin{cases}\prod_{j=1}^{\mu}\left(-\alpha_{j}\right)^{\ell_{j}\left(\ell_{j}-1\right) / 2} \prod_{1 \leq i<j \leq \mu}\left(\alpha_{i}-\alpha_{j}\right)^{\ell_{i} \ell_{j}}, & \mu \neq 1, \\ \left(-\alpha_{1}\right)^{\ell_{1}\left(\ell_{1}-1\right) / 2}, & \mu=1 .\end{cases}
$$




\section{$\S 4$. Proof of Lemma 3.3}

\section{§4.1. Elementary transformations of the matrix $\mathcal{A}$}

We prepare some notation. We put

$$
{ }^{t}\left(\left(\tau^{L-1}, \ldots, \tau^{0}\right) \mathcal{A}_{m}\left[L, \ell_{m}\right]\right)=\left(\begin{array}{c}
\sum_{p=0}^{L-1} \tau^{p} \Delta_{\alpha}^{p} f_{m}^{[1]} \\
\tau \sum_{p=0}^{L-2} \tau^{p} \Delta_{\alpha}^{p} f_{m}^{[2]} \\
\vdots \\
\tau^{\ell_{m}-1} \sum_{p=0}^{L-\ell_{m}} \tau^{p} \Delta_{\alpha}^{p} f_{m}^{\left[\ell_{m}\right]}
\end{array}\right)=: \vec{F}_{m}\left[\ell_{m}\right] .
$$

With this notation, we have

$$
\begin{aligned}
\left(\tau^{L-1}, \ldots, \tau^{0}\right) \mathcal{A} & =\left(\tau^{L-1}, \ldots, \tau^{0}\right)\left(\mathcal{A}_{1}\left[L, \ell_{1}\right], \ldots, \mathcal{A}_{\mu}\left[L, \ell_{\mu}\right]\right) \\
& =\left({ }^{t} \vec{F}_{1}\left[\ell_{1}\right], \ldots,{ }^{t} \vec{F}_{\mu}\left[\ell_{\mu}\right]\right) .
\end{aligned}
$$

Now we define the notation $\|\cdot\|$ so that the following equality holds:

$$
\mathcal{A}=\left\|{ }^{t} \vec{F}_{1}\left[\ell_{1}\right], \ldots,{ }^{t} \vec{F}_{\mu}\left[\ell_{\mu}\right]\right\|=\left\|\begin{array}{c}
{ }^{t} \\
\vec{F}_{1}\left[\ell_{1}\right] \\
\vdots \\
\vec{F}_{\mu}\left[\ell_{\mu}\right]
\end{array}\right\| .
$$

The definition should be understood from the following examples.

Example 4.1. 1. $\mathcal{A}=\left(\begin{array}{cc}1 & 1 \\ \beta & \alpha\end{array}\right)={ }^{t}\left\|\begin{array}{l}1 \cdot \tau+\beta \\ 1 \cdot \tau+\alpha\end{array}\right\|$.

2. $\mathcal{A}=\left(\begin{array}{ccc}1 & 1 & 1 \\ \alpha+\beta & \beta & 2 \alpha \\ \alpha \beta & 0 & \alpha^{2}\end{array}\right)=\left\|\begin{array}{c}t \\ \alpha \cdot \tau^{2}+(\alpha+\beta) \tau+\alpha \beta \\ \tau(1 \cdot \tau+\beta) \\ 1 \cdot \tau^{2}+2 \alpha \tau+\alpha^{2}\end{array}\right\|=\left\|\begin{array}{c}t \\ (\tau+\alpha)(\tau+\beta) \\ \tau(\tau+\beta) \\ (\tau+\alpha)^{2}\end{array}\right\|$.

3. The matrix

$$
\mathcal{A}=\left(\begin{array}{ccccc}
1 & 1 & \ldots & \ldots & 1 \\
\vdots & \vdots & & . \cdot & \\
\frac{(L-1)(L-2)}{2 !} \alpha^{L-3} & (L-2) \alpha^{L-3} & . . & & \\
(L-1) \alpha^{L-2} & \alpha^{L-2} & & & \\
\alpha^{L-1} & 0 & & & O
\end{array}\right)
$$

is represented as 


$$
{ }^{t}\left\|\begin{array}{c}
1 \cdot \tau^{L-1}+(L-1) \alpha \tau^{L-2}+\cdots+\alpha^{L-1} \\
\tau\left(1 \cdot \tau^{L-2}+(L-2) \alpha \tau^{L-3}+\cdots+\alpha^{L-2}\right) \\
\vdots \\
\tau^{L-2}(1 \cdot \tau+\alpha) \\
\tau^{L-1} \cdot 1
\end{array}\right\|=\left\|\begin{array}{c}
t \\
\tau(\tau+\alpha)^{L-2} \\
\vdots \\
\tau^{L-2}(\tau+\alpha) \\
\tau^{L-1}
\end{array}\right\|
$$

Further, we set

$$
\begin{gathered}
f[\tau+\alpha]=\prod_{j=1}^{\mu}\left(\tau+\alpha_{j}\right)^{\ell_{j}}=: \prod_{j=1}^{\mu} \hat{f}_{j}(\tau), \quad \hat{f}_{j}(\tau):=\left(\tau+\alpha_{j}\right)^{\ell_{j}}, \\
\check{f}_{m}(\tau):=f[\tau+\alpha] /\left(\tau+\alpha_{m}\right)^{\ell_{m}} \quad\left(\Leftrightarrow \check{f}_{m}(\tau) \hat{f}_{m}(\tau)=f[\tau+\alpha]\right) .
\end{gathered}
$$

With this notation, we have

$$
\sum_{p=0}^{L-n} \tau^{p} \Delta_{\alpha}^{p} f_{m}^{[n]}[\alpha]=f[\tau+\alpha] /\left(\tau+\alpha_{m}\right)^{n}=\left(\tau+\alpha_{m}\right)^{\ell_{m}-n} \check{f}_{m}(\tau),
$$

and

$$
\vec{F}_{m}\left[\ell_{m}\right]=\left(\begin{array}{c}
\left(\tau+\alpha_{m}\right)^{\ell_{m}-1} \check{f}_{m}(\tau) \\
\tau\left(\tau+\alpha_{m}\right)^{\ell_{m}-2} \check{f}_{m}(\tau) \\
\tau^{2}\left(\tau+\alpha_{m}\right)^{\ell_{m}-3} \check{f}_{m}(\tau) \\
\vdots \\
\tau^{\ell_{m}-2}\left(\tau+\alpha_{m}\right) \check{f}_{m}(\tau) \\
\tau^{\ell_{m}-1} \check{f}_{m}(\tau)
\end{array}\right) .
$$

Now, by elementary transformations of matrices, we can prove the following lemma.

\section{Lemma 4.2.}

$$
\operatorname{det} \mathcal{A}=\operatorname{det}\left\|\begin{array}{c}
\vec{F}_{1}\left[\ell_{1}\right] \\
\vdots \\
\vec{F}_{\mu}\left[\ell_{\mu}\right]
\end{array}\right\|=\prod_{j=1}^{\mu}\left(-\alpha_{j}\right)^{\frac{1}{2} \ell_{j}\left(\ell_{j}-1\right)} \times \operatorname{det}\left\|\begin{array}{c}
\check{\mathbf{F}}_{1}\left[\ell_{1}\right] \\
\vdots \\
\check{\mathbf{F}}_{\mu}\left[\ell_{\mu}\right]
\end{array}\right\|,
$$

where

$$
\check{\mathbf{F}}_{m}\left[\ell_{m}\right]=\left(\begin{array}{c}
\tau^{\ell_{m}-1} \check{f}_{m}(\tau) \\
\tau^{\ell_{m}-2} \check{f}_{m}(\tau) \\
\vdots \\
\tau \check{f}_{m}(\tau) \\
\check{f}_{m}(\tau)
\end{array}\right)
$$


Proof. For each $m$ we make the elementary transformations

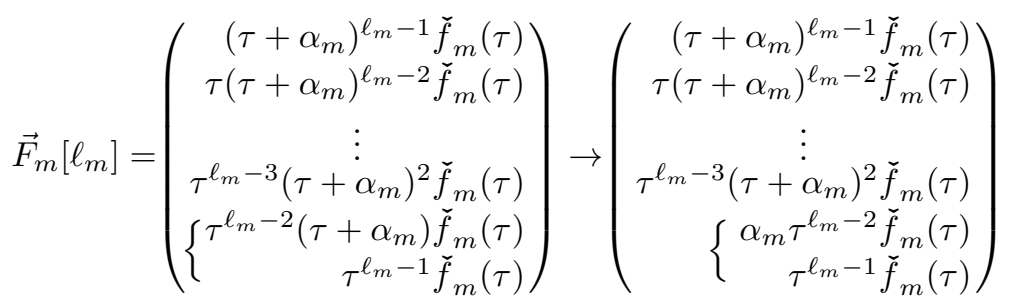

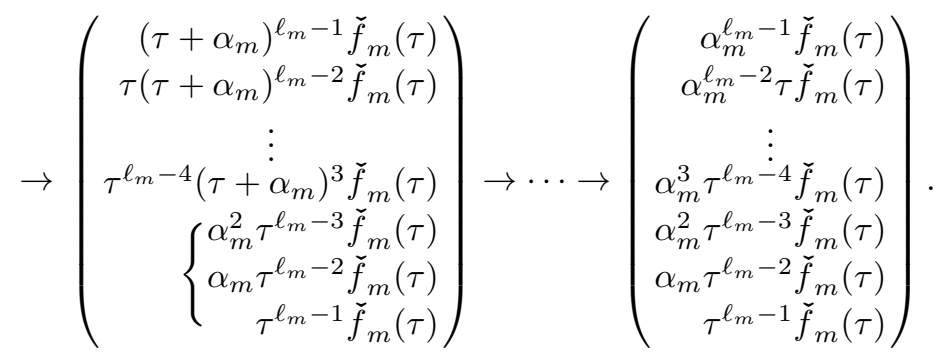

Since the determinant is invariant under these transformations, we get the formula (4.2), where we have changed the order of rows.

Thus the proof of Lemma 3.3 is reduced to showing

\section{Lemma 4.3.}

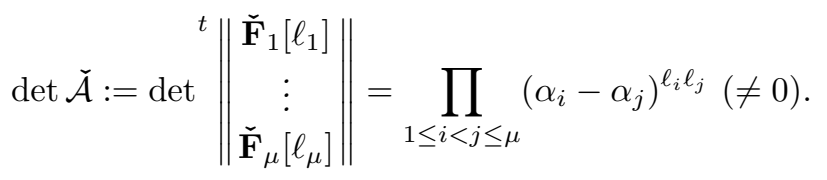

\section{\$4.2. Notation and lemmas}

In this subsection, we prepare additional notation and three lemmas about matrices. Let $p(\tau)=\sum_{j=0}^{\ell} p_{j} \tau^{j}$ and $k \geq 1$. We define a matrix $\ell+k M_{k}[p(\tau)]$ of size $(\ell+k) \times k$ by

$$
{ }_{\ell+k} M_{k}[p(\tau)]:=\left(\begin{array}{ccc}
p_{\ell} & & O \\
p_{\ell-1} & \ddots & \\
\vdots & & p_{\ell} \\
\vdots & & p_{\ell-1} \\
p_{0} & & \vdots \\
& \ddots & \vdots \\
O & & p_{0}
\end{array}\right)=\left\|\begin{array}{c}
\tau^{k-1} p(\tau) \\
\tau^{k-2} p(\tau) \\
\vdots \\
\tau p(\tau) \\
p(\tau)
\end{array}\right\|
$$


With this notation, we have

$$
\check{\mathcal{A}}=\left({ }_{L} M_{\ell_{1}}\left[\check{f}_{1}(\tau)\right], \ldots,{ }_{L} M_{\ell_{\mu}}\left[\check{f}_{\mu}(\tau)\right]\right),
$$

where $\check{f}_{m}(\tau)=f[\tau+\alpha] /\left(\tau+\alpha_{m}\right)^{\ell_{m}}=\prod_{j=1, j \neq m}^{\mu}\left(\tau+\alpha_{j}\right)^{\ell_{j}}$.

In the following, $p(\tau), q(\tau)$ and $r(\tau)$ are polynomials of degree $\ell, m$ and $n$, and their coefficients of $\tau^{j}$ are denoted by $p_{j}, q_{j}$ and $r_{j}$, respectively.

Lemma 4.4. Let $P(\tau)=p(\tau) r(\tau):=\sum_{k=0}^{\ell+n} P_{k} \tau^{k}$. Then

$$
{ }_{(\ell+n)+1} M_{1}[P(\tau)]={ }_{(\ell+n)+1} M_{1}[p(\tau) r(\tau)]={ }_{n+(\ell+1)} M_{\ell+1}[r(\tau)]_{\ell+1} M_{1}[p(\tau)],
$$

that is,

$$
\left(\begin{array}{c}
P_{\ell+n} \\
\vdots \\
P_{0}
\end{array}\right)=\left(\begin{array}{ccc}
r_{n} & & O \\
r_{n-1} & \ddots & \\
\vdots & & r_{n} \\
\vdots & & r_{n-1} \\
r_{0} & & \vdots \\
& \ddots & \vdots \\
O & & r_{0}
\end{array}\right)\left(\begin{array}{c}
p_{\ell} \\
\vdots \\
p_{0}
\end{array}\right)
$$

Proof. This is an immediate consequence of $P_{k}=\sum_{i+j=k} p_{i} r_{j}$.

Lemma 4.5. We have

$$
\begin{aligned}
\left({ }_{(\ell+n)+m} M_{m}[p(\tau) r(\tau)] \vdots{ }_{(m+n)+\ell} M_{\ell}[q(\tau) r(\tau)]\right) & \\
\quad={ }_{n+(\ell+m)} M_{\ell+m}[r(\tau)](\ell+m & \left.M_{m}[p(\tau)] \vdots{ }_{m+\ell} M_{\ell}[q(\tau)]\right)
\end{aligned}
$$

Moreover, $N:=\left({ }_{\ell+m} M_{m}[p(\tau)] \vdots{ }_{m+\ell} M_{\ell}[q(\tau)]\right)$ is a square matrix of size $\ell+m$, and its determinant is the resultant of the polynomials $p(\tau)$ and $q(\tau)$. Hence

$$
\operatorname{det} N=p_{\ell}^{m} q_{m}^{\ell} \prod_{1 \leq i \leq \ell, 1 \leq j \leq m}\left(x_{i}-y_{j}\right),
$$

where $\left\{x_{i}\right\}$ and $\left\{y_{j}\right\}$ are the roots of $p(\tau)$ and $q(\tau)$, respectively.

Proof. The formula (4.8) follows by applying Lemma 4.4. For the theory of resultants, we refer to [9].

The next lemma follows from Lemma 4.5. 
Lemma 4.6. Let $s(\tau)=\sum_{j=0}^{\ell+m} s_{j} \tau^{j}$. Then

$$
\begin{aligned}
\left({ }_{(\ell+m)+n} M_{n}[s(\tau)] \vdots{ }_{(\ell+n)+}\right. & \left.M_{m}[p(\tau) r(\tau)] \vdots{ }_{(m+n)+\ell} M_{\ell}[q(\tau) r(\tau)]\right) \\
= & \left({ }_{(\ell+m)+n} M_{n}[s(\tau)] \vdots{ }_{n+(\ell+m)} M_{\ell+m}[r(\tau)]\right) \\
& \times\left(E_{n} \oplus\left(\ell+m M_{m}[p(\tau)] \vdots m+\ell M_{\ell}[q(\tau)]\right)\right),
\end{aligned}
$$

where $E_{n}$ denotes the unit matrix of size $n$.

\section{§4.3. Proof of Lemma 4.3}

Lemma 4.3 is obtained by $(\mu-2)$-fold application of Lemma 4.6. Firstly, we recall

$$
\check{\mathcal{A}}=\left({ }_{L} M_{\ell_{1}}\left[\check{f}_{1}(\tau)\right], \ldots,{ }_{L} M_{\ell_{\mu}}\left[\check{f}_{\mu}(\tau)\right]\right),
$$

where $\check{f}_{m}(\tau)=f[\tau+\alpha] /\left(\tau+\alpha_{m}\right)^{\ell_{m}}=\prod_{j=1, j \neq m}^{\mu}\left(\tau+\alpha_{j}\right)^{\ell_{j}}$.

For brevity, set

$$
\begin{aligned}
L[\leq i]:=\sum_{j=1}^{i} \ell_{j}, \quad L[\geq i]:=\sum_{j=i}^{\mu} \ell_{j} \quad\left(L[\leq i]+L[\geq i+1]=L=\sum_{j=1}^{\mu} \ell_{j}\right), \\
f_{\leq m}(\tau):=\prod_{j=1}^{m}\left(\tau+\alpha_{j}\right)^{\ell_{j}}, \quad f_{\geq m}(\tau):=\prod_{j=m}^{\mu}\left(\tau+\alpha_{j}\right)^{\ell_{j}} \\
\left(f_{\leq m}(\tau) f_{\geq m+1}(\tau)=f[\tau+\alpha]\right) .
\end{aligned}
$$

We denote $\check{\mathcal{A}}=\mathcal{A}_{\mu}$.

Since the polynomials $\check{f}_{\mu-1}(\tau)$ and $\check{f}_{\mu}(\tau)$ have a common factor $f_{\leq \mu-2}(\tau)=$ $\prod_{j=1}^{\mu-2}\left(\tau+\alpha_{j}\right)^{\ell_{j}}$, the formula (4.10) implies that

$$
\begin{aligned}
\mathcal{A}_{\mu}= & \left({ }_{L} M_{\ell_{1}}\left[\check{f}_{1}(\tau)\right], \ldots,{ }_{L} M_{\ell_{\mu-2}}\left[\check{f}_{\mu-2}(\tau)\right] \vdots{ }_{L} M_{\ell_{\mu-1}}\left[\check{f}_{\mu-1}(\tau)\right] \vdots{ }_{L} M_{\ell_{\mu}}\left[\check{f}_{\mu}(\tau)\right]\right) \\
= & \left({ }_{L} M_{\ell_{1}}\left[\check{f}_{1}(\tau)\right], \ldots,{ }_{L} M_{\ell_{\mu-2}}\left[\check{f}_{\mu-2}(\tau)\right] \vdots{ }_{L} M_{L[\geq \mu-1]}\left[f_{\leq \mu-2}(\tau)\right]\right) \\
& \times\left(E_{L[\leq \mu-2]} \oplus\left({ }_{L[\geq \mu-1]} M_{\ell_{\mu-1}}\left[\hat{f}_{\mu}(\tau)\right] \vdots{ }_{L[\geq \mu-1]} M_{\ell_{\mu}}\left[\hat{f}_{\mu-1}(\tau)\right]\right)\right) \\
= & : \mathcal{A}_{\mu-1} \cdot\left(E_{L[\leq \mu-2]} \oplus \widehat{\mathcal{A}}(\mu, \mu-1)\right) .
\end{aligned}
$$

Here $\operatorname{det} \widehat{\mathcal{A}}(\mu, \mu-1)$ is the resultant of $\hat{f}_{\mu}(\tau)=\left(\tau+\alpha_{\mu}\right)^{\ell_{\mu}}$ and $\hat{f}_{\mu-1}(\tau)=$ $\left(\tau+\alpha_{\mu-1}\right)^{\ell_{\mu-1}}$, and its value is $\left(\alpha_{\mu-1}-\alpha_{\mu}\right)^{\ell_{\mu-1} \ell_{\mu}}$.

Secondly, since the polynomials $\check{f}_{\mu-2}(\tau)$ and $f_{\leq \mu-2}(\tau)$ have a common factor $f_{\leq \mu-3}(\tau)=\prod_{j=1}^{\mu-3}\left(\tau+\alpha_{j}\right)^{\ell_{j}}$, we have 


$$
\begin{aligned}
\mathcal{A}_{\mu-1} \\
=\left({ }_{L} M_{\ell_{1}}\left[\check{f}_{1}(\tau)\right], \ldots,{ }_{L} M_{\ell_{\mu-3}}\left[\check{f}_{\mu-3}(\tau)\right] \vdots{ }_{L} M_{\ell_{\mu-2}}\left[\check{f}_{\mu-2}(\tau)\right] \vdots{ }_{L} M_{L[\geq \mu-1]}\left[f_{\leq \mu-2}(\tau)\right]\right) \\
=\left({ }_{L} M_{\ell_{1}}\left[\check{f}_{1}(\tau)\right], \ldots,{ }_{L} M_{\ell_{\mu-3}}\left[\check{f}_{\mu-3}(\tau)\right] \vdots{ }_{L} M_{L[\geq \mu-2]}\left[f_{\leq \mu-3}(\tau)\right]\right) \\
\quad \times\left(E_{L[\leq \mu-3]} \oplus\left({ }_{L[\geq \mu-2]} M_{\ell_{\mu-2}}\left[f_{\geq \mu-1}(\tau)\right] \vdots{ }_{L[\geq \mu-2]} M_{L[\geq \mu-1]}\left[\hat{f}_{\mu-2}(\tau)\right]\right)\right) \\
=: \mathcal{A}_{\mu-2} \cdot\left(E_{L[\leq \mu-3]} \oplus \widehat{\mathcal{A}}(\geq \mu-1, \mu-2)\right) .
\end{aligned}
$$

Here, $\operatorname{det} \widehat{\mathcal{A}}(\geq \mu-1, \mu-2)$ is the resultant of $f_{\geq \mu-1}(\tau)=\left(\tau+\alpha_{\mu-1}\right)^{\ell_{\mu-1}}\left(\tau+\alpha_{\mu}\right)^{\ell_{\mu}}$ and $\hat{f}_{\mu-2}(\tau)=\left(\tau+\alpha_{\mu-2}\right)^{\ell_{\mu-2}}$, and its value is $\left(\alpha_{\mu-2}-\alpha_{\mu-1}\right)^{\ell_{\mu-2} \ell_{\mu-1}}$. $\left(\alpha_{\mu-2}-\alpha_{\mu}\right)^{\ell_{\mu-2} \ell_{\mu}}$.

By continuing these arguments, we finally get

$$
\operatorname{det} \check{\mathcal{A}}=\operatorname{det} \mathcal{A}_{\mu}=\prod_{k=1}^{\mu-1} \operatorname{det} \widehat{\mathcal{A}}(\geq \mu-k+1, \mu-k)=\prod_{1 \leq i<j \leq \mu}\left(\alpha_{i}-\alpha_{j}\right)^{\ell_{i} \ell_{j}},
$$

where $\widehat{\mathcal{A}}(\geq \mu, \mu-1)=\widehat{\mathcal{A}}(\mu, \mu-1)$ and $\mathcal{A}_{2}=\widehat{\mathcal{A}}(\geq 2,1)$.

\section{References}

[1] W. Balser, From divergent power series to analytic functions, Lecture Notes in Math. 1582, Springer, 1994. Zbl 0810.34046 MR 1317343

[2] W. Balser and M. Miyake, Summability of formal solutions of certain partial differential equations with constant coefficients, Acta Sci. Math. (Szeged) 65 (1999), 543-551. Zbl 0987.35032 MR 1737270

[3] K. Ichinobe, The Borel sum of divergent Barnes hypergeometric series and its application to a partial differential equation, Publ. RIMS Kyoto Univ. 37 (2001), 91-117. Z Zbl 0969.33004 MR 1815996

[4] Integral representation for Borel sum of divergent solution to a certain nonKowalevski type equation, Publ. RIMS Kyoto Univ. 39 (2003), 657-693. Zbl 1063.35049 MR 2025459

[5] D. Lutz, M. Miyake and R. Schäfke, On the Borel summability of divergent solutions of the heat equation, Nagoya Math. J. 154 (1999), 1-29. Zbl 0958.35061 MR 1689170

[6] M. Miyake, Borel summability of divergent solutions of the Cauchy problem to nonKowalevskian equations, in Partial differential equations and their applications (Wuhan, 1999), World Sci., 1999, 225-239. Zbl 0990.35005 MR 1742036

[7] M. Miyake and K. Ichinobe, Hierarchy of partial differential equations and fundamental solutions associated with summable formal solutions of a partial differential equations of non Kowalevski type, in Differential equations and asymptotic theory in mathematical physics (Wuhan, 2003), World Sci., 2004, 330-342. Zbl 1145.35355 MR 2161978

[8] S. Mizohata, Mathematical Analysis, Vol. 1, Chap. 4, Asakura Shoten, 1973 (in Japanese).

[9] T. Takagi, Lectures on Algebra, new edition, Chap. 10, Kyoritsu Shuppan, 1965 (in Japanese). 